\title{
Effects of epidermal growth factor on reduction of the formation of thrombus and vessel wall healing in an experimental rat model
}

\author{
Yumusak $\mathrm{N}^{1}$, Yavuz $\mathrm{U}^{2}$, Sarikaya $\mathrm{B}^{3}$, Yucel $\mathrm{G}^{4}$ \\ Department of Pathology, Faculty of Veterinary Medicine, University of Harran, Sanliurfa, Turkey. \\ nihatyumusak@harran.edu.tr
}

\begin{abstract}
OBJECTIVES: The aim of the current study was to investigate the synergistic effects of epidermal growth factor (EGF) and Enoxaparin in thrombus resolution.

METHOD: Forty rats were divided into five groups ( $n=8 /$ group). Thrombosis was induced in all groups except the Sham group. Group 1: Sham; Group 2: Phosphate buffered saline; Group 3: Enoxaparin; Group 4: EGF; Group 5: EGF+Enoxaparin. The treatments were applied 2 hours preoperatively, then postoperatively at 48 hours. Rats were sacrificed 7 days after the 2 nd injection. Tissue samples were examined with hematoxylineosin, trichrome, vascular endothelial growth factors (VEGF), von Villebrand factor (VWF), CD34 and CD68 for histopathological and immunohistochemical analyses.

RESULTS: Neovascularisation, recanalization and macrophage accumulation were statistically significantly higher in the EGF+Enoxaparin group than the other groups $(p<0.05)$, and the volume of thrombus was determined to be significantly lower. Recanalization was found to be higher in the Enoxaparin group than in the other groups. As for the thrombus resolution, statistically significant regress in the EGF+Enoxaparin group ( $p<$ 0.05 ) compared to the other groups was found. Immunohistochemical antibodies were statistically higher in the EGF+Enoxaparin group than in the other groups $(p<0.05)$.

CONCLUSION: The results of this study demonstrate that concomitant use of EGF and Enoxparin has a synergistic effect and contributes significantly to thrombus resolution (Fig. 10, Ref. 35). Text in PDF www.elis.sk. KEY WORDS: epidermal growth factor, histopathology, immunohistochemistry, thrombosis.
\end{abstract}

\section{Introduction}

Thrombosis is a complex disorder that occurs in a chain of events including platelet adhesion, activation and aggregation, which depend on genetic and environmental factors. In the formation of a thrombus, there are predisposing factors including cardiac malformation, acquired protein deficiency, polymorphism and genetic predisposition involved $(1,2)$. Causes leading to vascular endothelial injury include atherosclerosis, inflammation, catheterization in neonates, open operative complications and fractures of extremities, and these are the most important causes of thrombus formation $(3,4)$. Healthy vascular endothelial cells ensure haemostasis through anti-thrombotic and pro-thrombotic activities. This includes the release of mediators which suppress vascular dilatation and thrombin and control the increase of fibrin, as well as accelerating the removal of free radicals (5). As a result of trauma in the vascular endothelium, there is a decrease in

${ }^{1}$ Department of Pathology, Faculty of Veterinary Medicine, University of Harran, Sanliurfa, Turkey, ${ }^{2}$ Department of Surgery University of Harran, Faculty of Veterinary Medicine, Eyyubiye, Sanliurfa, Turkey, ${ }^{3}$ Department of Orthopedics and Traumatology, University of Harran, Faculty of Medicine, Sanliurfa, Turkey, and ${ }^{4}$ Department of Pathology University of Ankara, Faculty of Veterinary Medicine, Diskapi, Ankara, Turkey

Address for correspondence: N. Yumusak, University of Harran, Faculty of Veterinary Medicine, Eyyubiye, 63100 Sanliurfa, Turkey.

Phone: +90.4143183899 , Fax: +90.4143183922 the generation of anti-thrombin, fibrinogen, factors V and VIII, plasminogen activator inhibitor, thrombin, and thrombin-activated fibrinolysis inhibitor (6). Inhibition of the fibrinolytic system is ensured by plasminogen activator inhibitors and plasmin inhibitors. When this balance is impaired, intravascular thrombosis occurs (7). Depending on the localization and severity of thrombosis, it may cause morbidity and mortality in patients. Symptoms such as seizures, hemiparesis and coma may develop due to central nervous system thrombosis (8).

The aim of treatment of thrombosis is to prevent local spread and the formation of emboli, both proximally and distally, to stimulate and accelerate fibrinolysis, and to prevent recurrence and post-thrombotic complications (9). Nowadays, anticoagulants, anti-thrombocytics and thrombolytics are used for therapeutic use (10). Enoxaparin sodium is a well-known low molecular weight heparin that is routinely used to prevent postoperative thromboses (11). Heparin which is introduced subcutaneously or intraperitoneally decreases fibrin accumulation and thus, prevents adhesions and thrombus formation (12).

Epidermal growth factor (EGF) is a 53-amino acid polypeptide, which was first isolated from the submaxillary saliva glands of mice in the 1960s (13). EGF exhibits a potent mitogenic effect via tyrosine-kinase receptors on cell membranes. EGF has antiinflammatory, anti-apoptotic, neurotrophic and neuroprotective effects $(14,15)$. Furthermore, EGF accelerates the formation of granulation tissue via the stimulatory effect on growth and re- 
production of fibroblasts, keratinocytes and vascular endothelial cells. It enhances epithelisation and stimulates the formation of new vessels $(16,17)$. The wound healing activity of EGF occurs through the mitogenic effect of binding to specific cell membrane receptor (HER-1) and adhering particularly to epithelial tissue, fibroblasts and vascular endothelial cells $(13,18)$.

Enoxaparin is used in the treatment of thrombosis resulting from trauma-induced vascular endothelial injury, as a known anticoagulant and EGF has proven positive effects on wound healing. Therefore, the aim of this study was to investigate the potential synergic effects of enoxaparin and EGF on the formation of thrombus.

\section{Materials and methods}

\section{Animals}

The study included a total of 40 Wistar albino rats, each weighing 250-300 g and aged 9-10 weeks. The rats were given a 1-week period of adaptation to laboratory conditions before the study. The animals were kept in polypropylene cages in an environment of 21 $\pm 2{ }^{\circ} \mathrm{C}$ room temperature, humidity $65-70 \%$ and a 12 -hour light/ dark cycle. They were fed standard feed and water. All the experimental phases were conducted in accordance with the approval and supervision of the Local Ethics Committee on the breeding and use of national laboratory animals. All procedures were in compliance with the principles of the Declaration of Helsinki for laboratory animals (IRB Approval No.: 2017/002-01-04).

\section{Organization of the thrombosis model}

The thrombosis model in rats was organized as in previous studies (19). General anaesthesia was administered under aseptic conditions as intramuscular (IM) $90 \mathrm{mg} / \mathrm{kg}$ of ketamine and $10 \mathrm{mg}$ / $\mathrm{kg}$ of xylazine. The right posterior femoral artery of each rat was used for the thrombosis model. The vessel was exposed through the incision made in the region and freed from the surrounding tissue. A tampon impregnated with $20 \%$ Ferric Chloride $(\mathrm{FeCl})$ was topically applied, as specified in literature, on the vessel as shown in Figure 1. To prevent the FeCI causing any damage to surrounding muscular and nervous tissues, the tampon was surrounded with aluminium foil as shown in Figure 1A. When cyanosis was observed on the vessel (Fig. 1B), the tampon was removed and the wounds of the experimental animals were left for secondary healing following appropriate wound site closure.

\section{Study design}

The rats were randomly divided into 5 groups of 8 . With the exception of the Sham group (Group 1) the thrombosis model and treatment was applied to the other 4 groups as follows:. Group 1 Sham): No damage was induced.

Group 2 (PBS): $1 \mathrm{ml}$ of PBS was administered intraperitoneally (IP).

Group 3 (Enoxaparin): Subcutaneous (SC) injection of Enoxaparin Sodium (Clexane ${ }^{\circledR}$, Sanofi-Aventis) at a dose of $2 \mathrm{mg} / \mathrm{kg}$.

Group 4 (EGF): $1 \mathrm{ml}$ of EGF (Heberprot-P $\subset 75 \mu \mathrm{g}$ ) was administered IP.

Group 5 (EGF+Enoxaparin): Enoxaparin Sodium (Clexane $\AA$, Sanofi-Aventis) at a dose of $2 \mathrm{mg} / \mathrm{kg}$ was administered SC and 1 $\mathrm{ml}$ of EGF was administered IP.

The initial treatments were applied 2 hours preoperatively, then a second treatment was applied at 48 hours postoperatively. The rats were sacrificed on the 7 th day after the second treatment. The right femoral region, where the thrombosis was induced, was incised and the damaged vessel was dissected. Tissue samples were fixed in $10 \%$ neutral formaldehyde $(\mathrm{pH} \mathrm{7.2-7.4)} \mathrm{for} \mathrm{histopatho-}$ logical and immunohistochemical studies.

\section{Histopathological examination}

The fixed vascular tissues were soaked under running water to remove the formaldehyde and were then dehydrated with increasing grades of ethanol $(50 \%, 75 \%, 96 \%, 100 \%)$ in an automated tissue follow-up device. Xylol staining was applied and the samples were blocked in a paraffin embedding device. Of the slices 4 microns in thickness cut from these blocks, the first three slices and every tenth slice were transferred onto a microscope slide via Leica RM 2125 RT for examinations under light microscope. The prepared preparations were passed through a graded alcohol and xylol series and then stained with hematoxylin-eosin
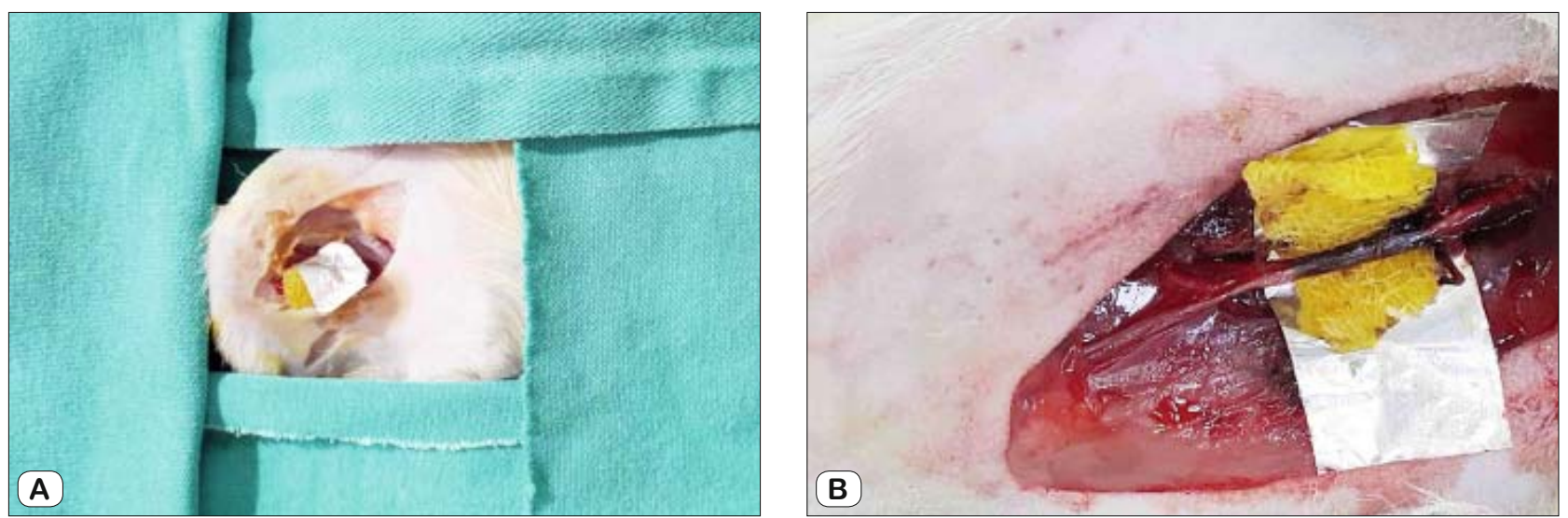

Fig. 1. Vessel injury site and developing thrombosis model. (A) - Vessel injury application by FeCI to femoral vessel. (B) - Color change site of FeCl3 injury 20-min after application. 

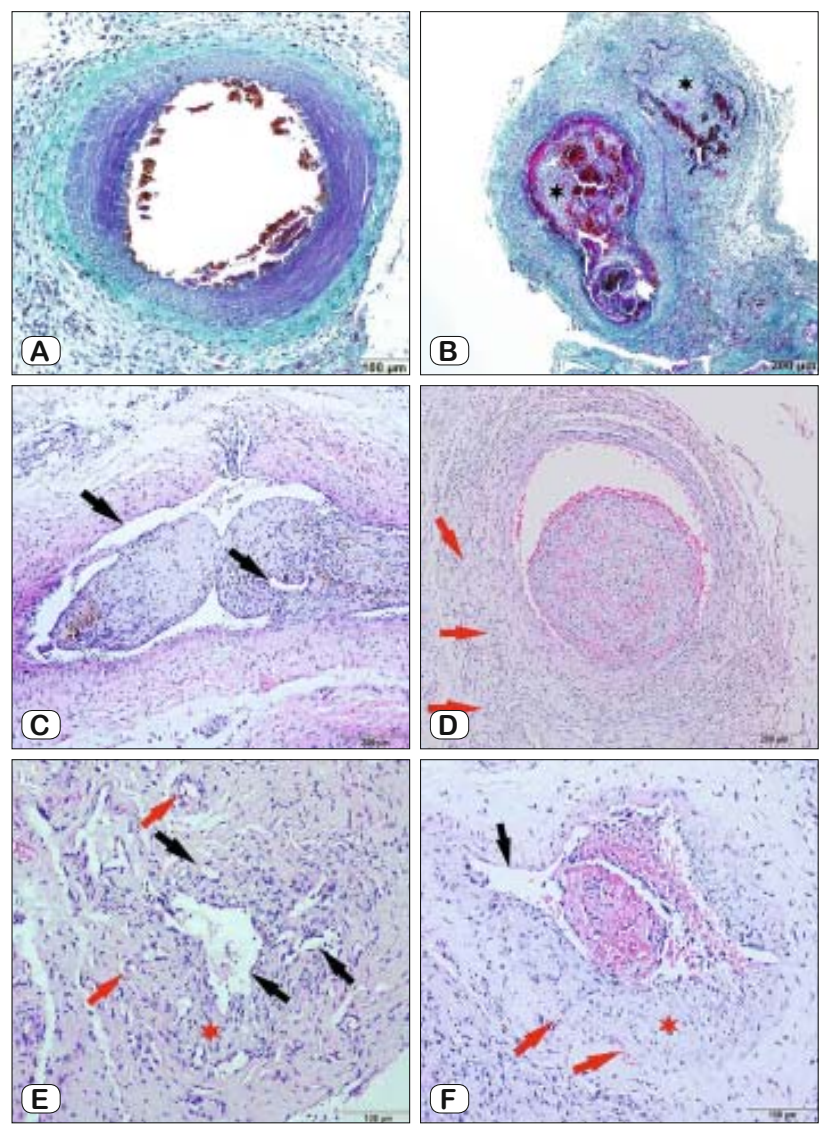

Fig. 2. Evaluation of histopathological changes, HE and trichrome staining. (A) - Sham group, with no vessel injury, normal vessel structure, trichrome staining. (B) - Control group, with injury and no treatment, occlusive thrombus in vessels (stars), trichrome staining. (C) Partial resolution of thrombi, formation of new channels around the vessel wall (arrows) and adjacent to lumen area in Enoxaparin group, HE staining. (D) - Formation of new capillary around the thrombi (red arrows) in EGF, HE staining. (E-F) - EGF+Enoxaparin group, near-complete resolution of the thrombi, new capillary formation (red arrows), new channels (black arrows) and recovery in the vessel wall (red stars), HE staining.

(HE) and trichrome staining. All samples were examined under a high-resolution light microscope (Olympus DP73 camera, Olympus BX53-DIC microscope; Tokyo, Japan) at x40 to x100 magnification for thrombosis wall diameter, neovascularisation localized around the thrombus and formation of new canals within the thrombus. The differences between the groups were evaluated.

\section{Immunohistochemical examination}

Neovascularisations around the thrombus were stained using vWF, VEGF and CD34 primary antibodies, to determine an increase in endothelial cells around the thrombus. CD68 primary antibody was used to determine macrophage intensity in this region, Accordingly, slices 4 microns in thickness were cut from the paraffin blocks and transferred onto Poly-L-lysine-coated microscope slides; after routine deparaffinization and rehydration methods. Staining was applied in accordance with the streptavidin-biotin-peroxidase complex (ABC) method (Zymed, Histostain Plus Kit, California, USA). For antigenic retrieval in tissues, heat treatment with citrate buffer ( $\mathrm{pH}$ 6.0) was applied in a microwave oven for 20 minutes. Endogenous peroxidise activation within the tissues was blocked for 10 minutes with 0.3 $\%$ hydrogen peroxide in methanol within $0.01 \mathrm{M}$ PBS. Prior to primary antibodies, the tissues were incubated with $5 \%$ normal goat serum for 30 minutes and protein blocking was performed. Then with incubation for 60 minutes, the slices were marked with anti-VWF (1 : 200; 36B11, Leica, Newcastle, UK), anti-VEGF (1 : 50; ab32152, Abcam, Cambridge, UK), CD68 (1 : 100; 514H12, Leica, Newcastle, UK) and CD34 (1 : 100; QBEnd/10, Leica, Newcastle, UK) primary antibodies under room temperature. Then the tissues were incubated for 30 minutes with rabbit antimouse biotinylated secondary antibody, and left for a further 30 mins incubation with streptavidin-peroxidase conjugate. In order to make the reaction visible, diaminobenzidine (DAB, Dako/ Denmark) chromogen was dropped onto the slices and left for 10 minutes. Finally, after staining with hematoxylin for posterior staining, the samples were soaked under running water. The tissues were passed through graded alcohol and xylol series and then adhered using Entellan. The whole staining procedure was conducted under room temperature and in humidity chambers. In the staining phases, PBS solution was used to soak the slices. The obtained data were evaluated under high-resolution microscope (Olympus DP73 camera, Olympus BX53-DIC microscope; Tokyo, Japan). For each sample, immunopositive cells determined at x40 and x100 magnifications were counted, recorded and then statistically evaluated.

\section{Statistical analysis}

All data analyses were applied using SPSS vn 24.0 for Windows software. Conformity to normal distribution of the data was tested using the Shapiro-Wilk Test. In the comparison of variables without normal distribution in more than two groups, the Kruskal Wallis test and Dunn's multiple comparison tests were used. Median $(25-75 \%)$ values were used as descriptive statistics. A value of $\mathrm{p}<0.05$ was accepted as statistically significant.

\section{Results}

\section{Histopathological results}

A statistically significant histological level of regression in thrombus resolution in the EGF + Enoxaparin group compared to the control and other groups was also statistically determined on figures $(p<0.05)$ (Fig. 2). The volume of thrombus was significantly lower in the EGF+Enoxaparin group compared to the other groups $(p<0.05)$ (Fig. 3). A significant regression in volume of thrombus was determined in the Enoxaparin group compared to the EGF and control groups ( $<<0.05)$ (Fig. 4). Recanalization formations were found to be significantly higher in the EGF+Enoxaparin group compared to the other groups $(\mathrm{p}<0.05)$. Recanalization formations were significantly higher in the Enoxaparin thrombosis group compared to the EGF and control groups $(p<0.05)$ (Fig. 5). Neovascularizations were at the highest level 

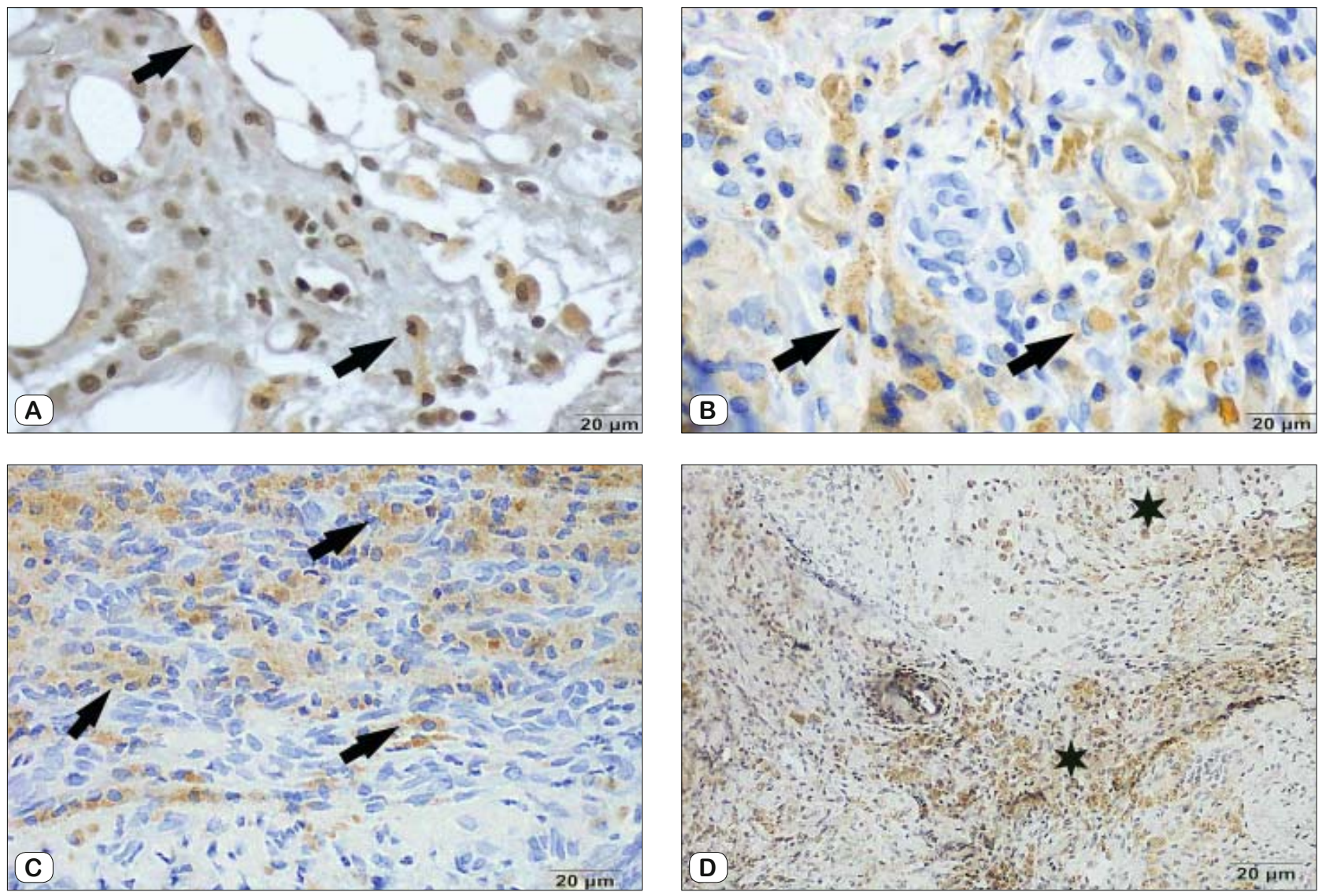

Fig. 3. immunohistochemical expression in thrombus EGF+Enoxaparin group for identification of neovascularization and macrophage accumulation: Immunopositive cells for VEGF (A), VWF (B), CD34 (C) (arrows) and CD68 (D) (stars) antibodies around the thrombi.

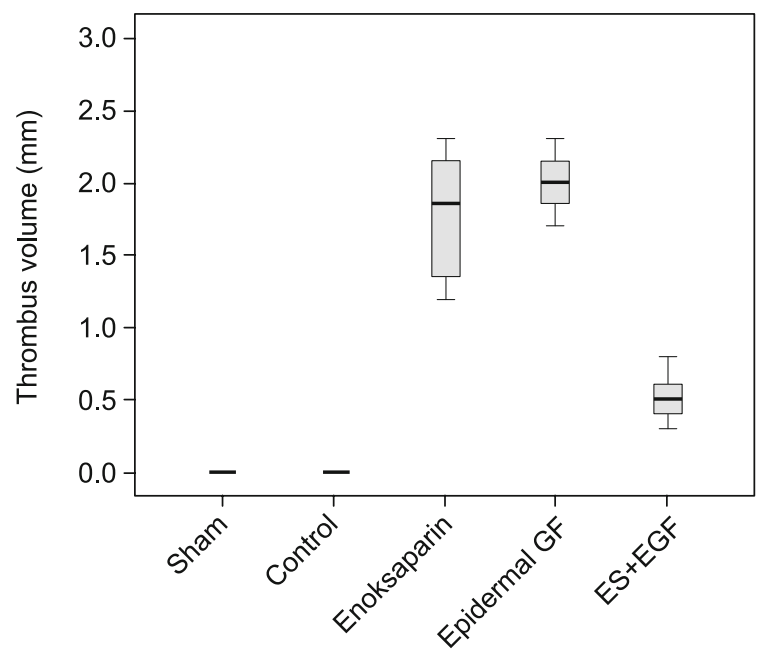

Fig. 4. Thrombus volume at day 10 after the procedure in rats treated groups.

in the EGF+ Enoxaparin group compared to the other groups $(\mathrm{p}<$ $0.05)$ and statistically significantly higher in the EGF group compared to the control group $(\mathrm{p}<0.05)$. No neovascularisation was determined in the Enoxaparin group ( $p>0.05)$ (Fig. 6).

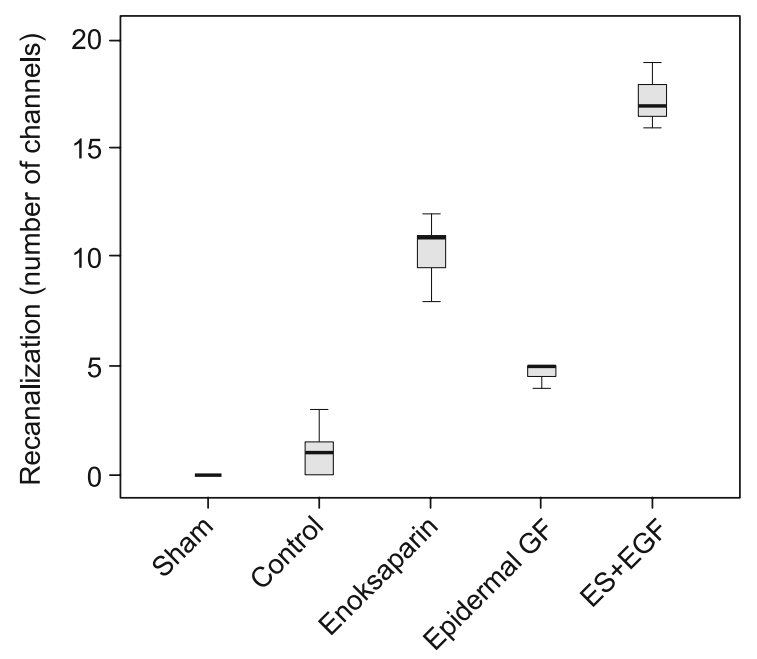

Fig. 5. Recanalisation (number of channels per field, 100x) after the procedure in rats treated groups.

\section{Immunohistochemical results}

Immunohistochemically, in the VEGF and vWF staining procedures, immunoreactive cell intensity determined around the thrombus was determined to be at a significantly high level in rats 
$752-758$

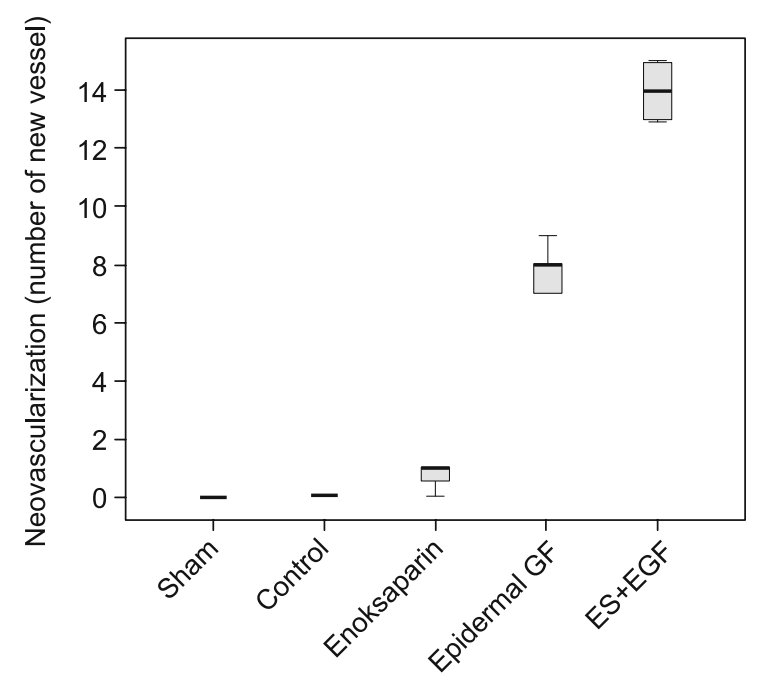

Fig. 6. Neovascularization (channels per field, 100x) after the procedure in rats treated groups.

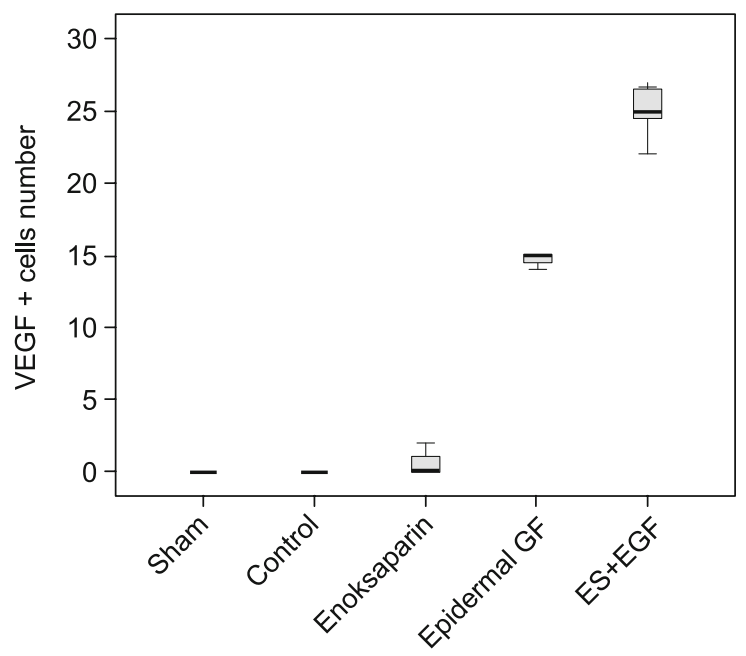

Fig. 7. Comparison of five groups for mean VEGF immunopositive cell numbers (per field, 100x).

treated with EGF+Enoxaparin compared to the EGF only group $(p<0.05)$ (Fig. 3A). VEGF-positive cell intensity was determined to be statistically significantly higher in the EGF+ Enoxaparin group compared to the other groups $(p<0.05)$ (Fig. 7). vWFimmunopositive cell intensity was observed to be statistically significantly higher in the EGF+ Enoxaparin group compared to the other groups $(\mathrm{p}<0.05)$ (Fig. 3B, Fig. 8). CD 34 antibody-positive cell intensity was observed to be statistically significantly higher in the EGF+ Enoxaparin group compared to the other groups $(\mathrm{p}<$ 0.05) (Fig. 3C, Fig. 9). Macrophage accumulation (CD68) around the thrombus was determined to be statistically significantly higher in the EGF+Enoxaparin group compared to the Enoxaparin only and control groups $(\mathrm{p}<0.05)$ (Fig. 3D, Fig. 10), and no statistical significance was found between the EGF+Enoxaparin and EGF treatment groups $(\mathrm{p}>0.05)$.

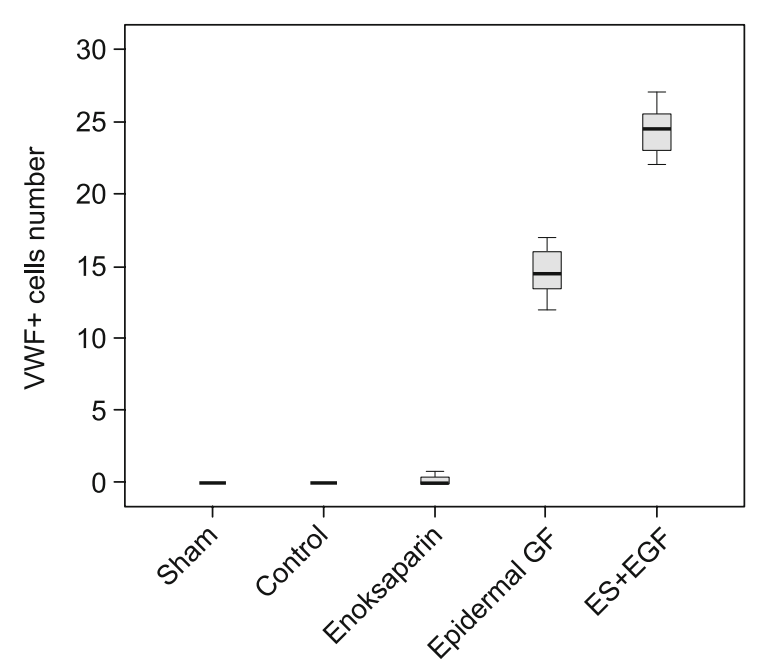

Fig. 8. Comparison of five groups for mean VWF immunopositive cell numbers (per field, 100x).

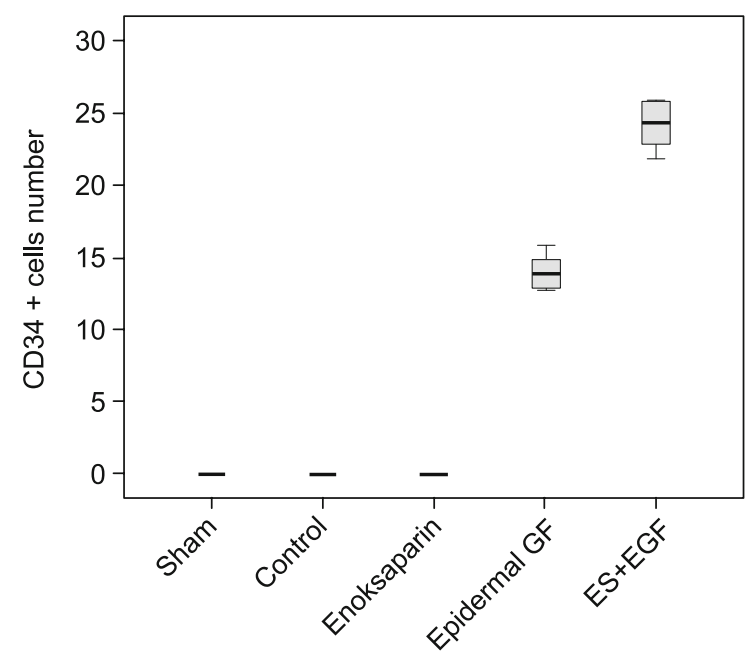

Fig. 9. Comparison of five groups for mean CD34 immunopositive cell numbers (per field, 100x).

\section{Discussion}

Thrombosis is an important complication which occurs from damage developing in the vessel wall and may be life-threatening. Anti-thrombotics or anticoagulants are used to prevent the potential formation of a thrombus and for treatment of thrombosis, (20, 21). The aim of thrombosis treatment is to prevent local spread and the formation of emboli, and to increase the recanalization rate by accelerating the organization of a thrombus (22). Thrombotic medications are plasminogen activators which catalyze conversion to plasmin, which is involved in the degradation of endogenous plasminogen into fibrin (10). Fibrinolytic treatment, however, occurs through degradation of fibrin, which is the major component in a clot, by plasmin which is activated through intravenous, intra-arterial or direct administration of plasminogen activators into the clot 


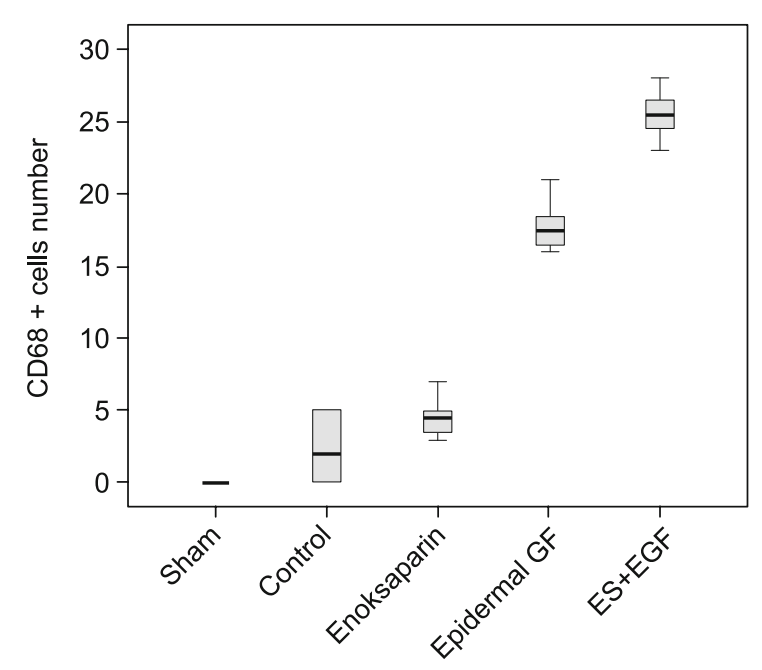

Fig. 10. Comparison of five groups for mean CD68 immunopositive cell numbers (per field, 100x).

(23). In a previous study, it was observed that Enoxaparin sodium, which is a low molecular weight heparin component with proven effectiveness, promoted the formation of new canals by accelerating thrombus resolution with a fibrinolytic effect $(9,11)$. It was determined that when Enoxaparin was used alone, the number of canals increased and the volume of the thrombus regressed, although neovascularisation did not occur. In addition, it was noticeable that when used with EGF, there was both a marked increase in the formation of canals and a significant reduction in thrombus volume.

In recent studies, it has become obvious that various new approaches have been sought for the preservation and treatment of thrombosis. Varma et al (24) reported in an experimental study that there was a significant increase in numbers of recanalization in basic fibroblast growth factor (bFGF) treatment but it had no effect on the organization of thrombus. Waltham et al (25) reported that VEGF treatment enhanced neovascularisation and recanalization, and helped regression of thrombus. Previous studies have determined that defibrotid triggers the formation of new canals and reduces the volume of thrombus via a fibrolytic effects in thrombosis (19). In the current study, however, with concomitant application of EGF and Enoxaparin, both healing of the vascular injury through repair and the prevention of recurrence of thrombosis were ensured, and a double-sided treatment was applied ensuring rapid resolution of the thrombus. In a similar experimental rat model study by Dilli et al (19) it was reported that with stem cell treatment, there was an increase in numbers of recanalizations and neovascularisations of thrombus and a significant reduction of the thrombus volume.

Organization in tissue damage has a complex structure involving various factors. Fibroblast activation, macrophage accumulation and endothelial cell proliferation in particular have important roles in wound healing $(26,27,28)$. Endothelium, fibroblast and smooth muscle cells have receptors secreted by EGF. These receptors exhibit a chemotactic effect by stimulating DNA synthesis on these cells, as well as accelerating wound healing by ensuring stimulation of angiogenesis and collagenesis $(16,17)$. EGF applications are successful especially in the treatment of wounds because of the stimulatory effect on growth and reproduction of fibroblasts, keratinocytes and vascular endothelial cells. Tarnawski and Jones (29) determined positive effects of EGF in the healing of ulcers which develop in the gastrointestinal system. Procaccino et al (30) also determined that experimentally, EGF reduced inflammation and accelerated mucosal repair in the healing of colitis and duodenal ulcers. Tuyet et al (31) reported that EGF application accelerated vascularisation and had positive effects on wound healing in diabetic patients where the healing of foot wounds is particularly challenging. Kennedy et al (14) reported that EGF suppressed renal tubular apoptosis following ureteral obstruction. It has been determined that EGF enhances healing and regeneration in ischemic renal injury induced in experimental rats $(32,33)$.

Many studies have also shown the positive effects through stimulation of epithelisation and vascularisation of EGF in burn injury treatment $(16,17,28,30)$. EGF has effects on wound healing with its inflammatory, fibroblastic activation and remodelling in all cases of tissue damage $(28,30)$. The organization and resolution of a thrombus which has formed as a result of a vascular injury has the characteristics of general wound healing (34). In the current study, it was also observed that EGF has a healing effect on vessels and on neovascularisation. In the literature, there are few studies on the effect of EGF on vessels. In one study, it was shown that EGF enhanced healing in the damaged artery and thereby exhibited a vasoprotective effect. In another study which examined neovascularisation it was demonstrated that EGF promoted angiogenesis in the same way as VEGF and FGD (35).

The current study results, in agreement with the findings of previous studies determined that EGF enhanced neovascularisation and the organization of thrombus, in a similar way to wound healing and the vessel wall injury was repaired by promoting macrophage accumulation. The current study also showed that neovascularization, which is one of the important markers of wound healing in particular, significantly increased around the thrombus together with both histological and immunohistochemical markers. It was also determined that in the group with significant organization of thrombus, macrophage accumulation was immunohistochemically strong and CD68 antibody-immunopositive cells were present at the site of the thrombus. Furthermore, with the well-known thrombolytic effect of Enoxaparin, a reduction in volume of the thrombus and an increase in the number of new canals were observed.

In conclusion, the effectiveness of EGF in the treatment of thrombosis was recognized in this study. This histopathological and immunohistochemical study conducted with EGF revealed that EGF accelerated healing in the thrombosed vessel wall, triggered neovascularisation and was therefore effective in organization of the thrombus. It was also determined that when used concomitantly with Enoxaparin, which is an anticoagulant agent, a synergistic effect occurred and thrombus resolution was accelerated. All these findings indicate that EGF may be used in the treatment of thrombosis. 
752-758

\section{Learning points}

- Epidermal growth factor reduced the harmful effects of thrombosis.

- Vessel wall healing activities are likely to be involved in the mechanism underlying the formation of thrombus.

- Epidermal growth factor can be considered for use as a wound healing agent for thrombosis.

\section{References}

1. Passamonti F, Rumi E, Pungolino E et al. Life expectancy and prognostic factors for survival in patients with polycythemia vera and essential thrombocythemia. Am J Med 2004; 117: 755-761.

2. Voetsch B, Joseph L. Genetic determinants of arterial thrombosis. Arteriosclerosis, thrombosis and vascular biology 2004; 24: 216-229.

3. Rauch U, Osende I, Fuster V et al. Thrombus formation on atherosclerotic plaques: pathogenesis and clinical consequences. Ann Intern Med 2001; 134: 224-238.

4. Rosa A, Cipollone L, Bertazzoni G. Venous thrombosis in emergency department: diagnosis, treatment, and disposition. Eur Rev Med Pharmacol Sci 2012; 16: 48-56.

5. Becker BF, Heindl B, Kupantt C et al. Endothelial function and hemostasis. Z Kardiol 2000; 89: 160-167.

6. Hass S. Venous thromboembolism in medical patients. The scope of the problem. Sem Thromb Hemost 2003; 29: 17-21.

7. Andrews RK, Gardiner EE, Shen Y, Berndt MC. Platelet interactions in thrombosis. IUBMB Life. 2004; 56: 13-18.

8. Wolanskyj AP, Schwager SM, McClure RF, Larson DR, Tefferi A. Essential thrombocythemia beyond the first decade: life expectancy, long term complication rates, and prognostic factors. Mayo Clin Proc 2006; 81: 159-166.

9. Comerota AJ, Paolini D. Treatment of acute iliofemoral deep venous thrombosis: a strategy of thrombus removal. Eur J Vasc Endovasc Surg 2007; 33: 351-360.

10. Kearon C, Kahn SR, Agnelli G et al. Antithrombotic therapy for venous thromboembolic disease: American college of chest physicians evidence-based clinical practice guidelines. Chest 2008: 454-545.

11. Mutlu AE, Boztosun A, Sumer D, Yank A. Comparison of efficiency of clexaneò "enoxaparin sodium" and methylene blue in prevention of adhesions after cesarean section in pregnant rats. J Turk Soc Obstet Gynecol 2012; 9: 216-223.

12. Dzupina A, Wald M, Lee BB, Stvrtinova V. Treatment of lower extremity superficial vein thrombosis with low molecular weight heparin. Bratisl Med J 2016; 117; 595-600.

13. Cohen S. Isolation of a mouse submaxillary gland protein accelerating incisor eruption and eyelid opening in the new-born animal. J Biol Chem 1962; 237: 1555-1562.

14. Kennedy WA, Buttyan R, Garcia-Montes E, D’Agati V, Olsson CA, Sawczuk IS. Epidermal growth factor suppresses renal tubular apoptosis following ureteral obstruction. Urology 1997; 49: 973-980.

15. Yarim GF, Kazak F. Epidermal Growth Factor. Kocatepe Vet J 2016; 93: $215-225$.

16. Su Z, Ma H, Wu Z, Zeng $\mathrm{H}$ et al. Enhancement of skin wound healing with decellularized scaffolds loaded with hyaluronic acid and epidermal growth factor. Mater Sci Eng C Mater Biol Appl 2014; 44: 440-448.
17. Wu Z, Tang Y, Fang H, Su Z et al. Decellularized scaffolds containing hyaluronic acid and EGF for promoting the recovery of skin wounds. J Mater Sci Mater Med. 2015; 26: 1-10.

18. Bazley LA, Gullick WJ. The epidermal growth factor receptor family. Endocr Relat Cancer 2005; 12: 17-27.

19. Dilli D, Kılıc E, Yumusak $\mathbf{N}$ et al. Additive effect of mesenchymal stem cells and defibrotide in an arterial rat thrombosis model. Archivos argentinos de pediatria, 2017; 115: 249-256.

20. Bachleitner-Hofmann T, Grumbeck E, Gisslinger H. Oral anticoagulants as secondary prophylaxis of thrombosis in patients with polycythemia vera: a retrospective analysis of 15 patients. Thromb Res 2003; 112: 229-32.

21. Long E, Pitfield AF, Kissoon N. Anticoagulation therapy: indications, monitoring, and complications. Pediatr Emerg Care 2011; 27: 55-61.

22. Evans CE, Humphries J, Mattock $K$ et al. Hypoxia and upregulation of hypoxia-inducible factor $1 \alpha$ stimulate venous thrombus recanalization. Arteriosclerosis, thrombosis and vascular biology 2010: 30; 2443-2451.

23. Lip GYH, Blann AD. Thrombogenesis and fibrinolysis in acute coronary syndromes. J Am Coll Cardiol 2000; 36: 2044-2046.

24. Varma MR, Moaveni DM, Dewyer NA, Varga AJ et al. Deep vein thrombosis resolution is not accelerated with increased neovascularization. J Vasc Surg 2004; 40: 536-542.

25. Waltham M, Burnand K, Fenske C, Modarai B, Humphries J, Smith A. Vascular endothelial growth factor naked DNA gene transfer enhances thrombus recanalization and resolution. J Vasc Surg 2005; 42: 1183-1189.

26. Modarai B, Burnand KG, Humphries J, Waltham M, Smith A. The role of neovascularisation in the resolution of venous thrombus. Thromb Haemost. 2005; 93: 801-809.

27. Velnar T, Bailey T, Smrkolj V. The wound healing process: an overview of the cellular and molecular mechanisms. J Int Med Res 2009; 37 : $1528-1542$

28. Young A, McNaught, CE. The physiology of wound healing. Surgery 2011; 29: 475-479.

29. Tarnawski A, Stachura J, Durbin T, Sarfeh IJ, Gergely H. Increased expression of epidermal growth factor receptor during gastric ulcer healing in rats. Gastroenterology 1992; 102: 695-698.

30. Procaccino F, Reinshagen M, Hoffmann P et al. Protective effect of epidermal growth factor in an experimental model of colitis in rats. Gastroenterology 1994; 107: 12-17.

31. Tuyet HL, Quynh TTN, Vo Hoang Minh $\mathbf{H}$ et al. The efficacy and safety of epidermal growth factor in treatment of diabetic foot ulcers: the preliminary results. Int Wound J 2009; 6: 159-166.

32. Caballero ME, Calunga J, Barber E, Cruz E, López-Saura P, Boix E, Berlanga J. Epidermal growth factor-mediated prevention of renal ischemia/reperfusion injury. Biotecnol Apl 2000; 17: 161-165.

33. Humes HD, Cieslinski DA, Coimbra TM, Messana JM, Glavao C. Epidermal growth factor enhances renal tubule cell regeneration and repair and accelerates the recovery of renal failure. J Clin Invest 1989; 84: $1757-1761$.

34. Wakefield TW, Myers DD, Henke PK. Mechanisms of venous thrombosis and resolution. Arterioscler Thromb Vasc Biol 2008; 28: 387-391.

35. Möller B, Rasmussen C, Lindblom B, Olovsson M. Expression of the angiogenic growth factors VEGF, FGF-2, EGF and their receptors in normal human endometrium during the menstrual cycle. Mol Hum Reprod. 2001; 7: 65-72.

Received June 13, 2017. Accepted July 7, 2017. 